We try to publish authors' responses in the same edition with readers' comments. Time constraints might prevent this in some cases. The problem is compounded in a bimonthly journal where continuity of comment and redress are difficult to achieve. When the redress appears 2 months after the comment, 4 months will have passed since the article was published. Therefore, we would suggest to our readers that their correspondence about published papers be submitted as soon as possible after the article appears.

\section{Pediatric Admissions by Family Physicians and Pediatricians}

To the Editor: The report by Bertolino and Gessner ${ }^{1}$ describing the experience of family physicians with pediatric inpatient care in a semirural hospital care raises a number of important issues concerning the role of family physicians in providing hospital care for children. They report on a cadre of 31 family physicians and 9 pediatricians providing inpatient care for hospitalized infants and children (excluding normal newborns) in a semirural hospital. By extrapolating from the data provided, I assume that family physicians in this study discharged, on average, 8 pediatric inpatients per year ( 6.6 infants and children, 1.4 sick newborns). Pediatricians working in the same hospital during the study period served as attending physicians for an average of 51 pediatric inpatients annually (33 infants and children, 18 sick newborns), and provided inpatient consultations to an additional 5 patients per year.

The pediatric inpatient experience reported in this study is similar to that of other family physicians in rural hospitals. ${ }^{2}$ In other settings, however, the typical family physician might have even fewer opportunities to care for hospitalized children. A study of 181,500 discharges of patients younger than 18 years old from urban and rural hospitals in Washington State showed that after excluding healthy newborns, the median annual number of discharges was 25 for pediatricians and 3 for family physicians. ${ }^{3}$ Even though most hospitalized children under the care of family physicians will have one of a limited number of common diagnoses, low volumes mean that it is unlikely that these physicians will treat the same condition twice in 1 year. Given these practice characteristics, can we justify training family physicians in pediatric inpatient care with the expectation that they provide this care for their patients once in practice?

There are a number of compelling reasons why training family physicians in pediatric inpatient care is important. The hospital environment provides exposure to a group of children very different from those seen in a well-child setting, and inpatient experience helps ensure that family physicians can recognize and stabilize sick children. The competency list suggested by Bertolino and Gessner forms a rational basis for developing training goals and objectives. Inpatient training also prepares family physicians to provide inpatient care for children under circumstances (common in rural practice) where pediatricians are unavailable or too few to provide adequate coverage for inpatient services.

The controversial question raised by the study of Bertolino and Gessner is whether family physicians practicing in hospitals with pediatricians should serve as inpatient attending physicians for hospitalized children. Although this study supports the notion that it is appropriate for pediatricians to serve as consultants to family physicians rather than as attending physicians for moderately ill children, some would argue that it is reasonable that pediatricians, by virtue of specialized training and considerably greater experience in inpatient care, are the logical providers of hospital care in these settings.

Some studies have suggested that the outcomes of inpatient care of adults by internists and family physicians are comparable., 5 The proportion of outpatient care dedicated to adults and the high volumes of inpatient care provided by family physicians to adults, however, are more likely to ensure that inpatient skills among family physicians for these patients are maintained. While few studies have compared the quality of inpatient care of children by pediatricians and family physicians, variation in the management of common problems that result in hospitalization (such as the care of the febrile infant ${ }^{6,7}$ suggests there might also be differences in outcomes of pediatric inpatient care as provided by pediatricians and family physicians.

As we seek to develop a rational policy for educating and deploying physicians to care for hospitalized children in a variety of settings, studies addressing the outcomes of hospital care as provided by family physicians and pediatricians should be undertaken. In the absence of data showing any clear difference in outcomes, models of care that promote collaboration between pediatricians and family physicians, such as described by Bertolino and Gessner, should be encouraged and supported.

Sanford M. Melzer, MD

Children's Hospital and Regional Medical Center

Department of Pediatrics

University of Washington School of Medicine, Seattle

\section{References}

1. Bertolino JG, Gessner TP. Pediatric admissions by family physicians and pediatricians in a semirural environment; implications for residency training. J Am Board Fam Pract 1999;12:128-32.

2. Melzer SM, Grossman DC, Hart LG, Rosenblatt RA. Hospital services for rural children in Washington State. Pediatrics 1997;99:196-203. 
3. Melzer SM, Grossman DC, Rivara FP. Physician experience with inpatient care in Washington State. Pediatrics 1999;97:65-70.

4. McGann KP, Bowman MA. A comparison of morbidity and mortality for family physicians' and internists' admissions. J Fam Pract 1990;31:541-5.

5. Hainer BL, Lawler FH. Comparison of critical care provided by family physicians and general internists. JAMA 1998;15:354-8.

6. Zerr DM, Del Beccaro MA, Cummings P. Predictors of physician compliance with a published guideline on management of febrile infants. Pediatr Infect Dis J 1999;18: $232-8$.

7. Wittler RR, Cain KK, Bass JW. A survey about management of febrile children without source by primary care physicians. Pediatr Infect Dis J 1998;17:271-7.

The above letter was referred to the author of the article in question, who offers the following reply.

To the Editor: I have reviewed the letter by Dr. Melzer and, overall, find it supportive of our conclusions.

Summarizing our work on a different level, we have been able to show that pediatricians are an important component of family practice training. When the training occurs in an environment of cooperation where there is a need for both types of providers, the result to the community is a family physician who is highly capable of providing care to most hospitalized children.
John G. Bertolino, MD, MSPH

Family Practice Residency, Latrobe Area Hospital

Department of Family Medicine Jefferson Medical College, Philadelphia, $\mathrm{Pa}$

\section{Severe Guillain-Barré Syndrome Associated with Campylobacter jejunt Infection}

To the Editor: I would like to point out an important, recently published article: "Quinolone-Resistant Campylobacter jejuni Infections in Minnesota, 1992 to 1998" (Smith Kirk E, Besser JM, Hedberg CW, et al. N Engl J Med 1999;340:1525-32). It is frightening that the Food and Drug Administration did not ban the use of quinolones in animals used for food in 1992. Even though the FDA banned their use in 1997, enrofloxacin was licensed for use in beef cattle in 1998.

On a recent visit to Canada in early May of this year, I contracted a Campylobacter infection that was resistant to ciprofloxacin. Because of the potential serious complications of Campylobacter infection in humans, I strongly concur with the conclusion: "A well-coordinated international program is needed to assess worldwide use of antibiotics in animals used for food and to ensure appropriate limitations of such use if it is shown to be deleterious to human health."

One wonders whether any decisions are made for long-term health, or do short-term profits (eg, feed lot cattle and crowded poultry farms) always win out?

Dorothyann $M$. Lindes, MD

Southwest Family Physicians Cleveland, Ohio 